\title{
Law of the Vital Few: Choosing Variables of Disease Activity in Rheumatoid Arthritis
}
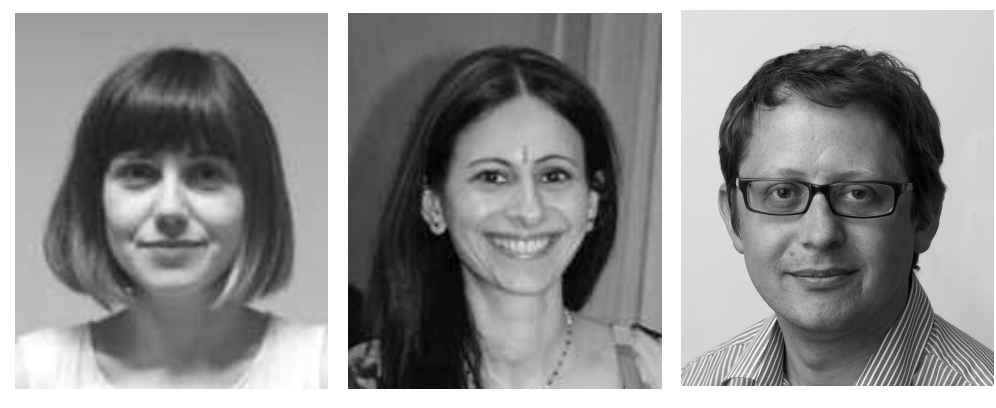

The Italian economist Vilfredo Pareto noticed that $20 \%$ of the pea pods in his garden contained $80 \%$ of the peas. His "Law of the Vital Few" seems to describe a wide variety of situations where $80 \%$ of the observed effect can be explained by $20 \%$ of the causes. The treatment of rheumatoid arthritis (RA) was revolutionized in the 1980s and 1990s by a small number of simple but powerful advances. Robust trials demonstrated the value of cheap, readily available drugs such as methotrexate. Simple clinical outcome measures such as the Disease Activity Score (DAS) were validated. Because our goals have since evolved to focus on smaller numbers of patients with resistant disease or poor prognosis or to personalize therapy, more complex and expensive techniques and interventions seem to be required to achieve better outcomes.

The longterm goal of therapy in RA is the preservation of function and prevention of joint damage. Treat-to-target trials such as TICORA demonstrate that both doctors and patients underestimate how much therapy is needed to achieve this ${ }^{2}$. Not surprisingly, perhaps, more therapy leads to less inflammation and better short-term and longterm outcomes. Current "best practice" in early RA is based on the use of composite scores such as the DAS or its 28-joint version (DAS28) to direct escalation of therapy ${ }^{3,4}$. However, while DAS-based targets might successfully encourage the use of more immunosuppressive drugs, this may be at the cost of reduced specificity, because of the limitations of clinical evaluation. Increasing treatment on a population level leads to population-level benefits, but individual patients may be either undertreated or overtreated.

The most obvious limitation of the DAS is that tender joint count and patient visual analog scales (VAS) may reflect other pathologies and patient states, including osteoarthritis, secondary damage, psychological factors, and fibromyalgia ${ }^{5,6}$. The DAS was first derived in early arthritis clinics in an era of ineffective therapies, when most sympto- matic patients had some active inflammation. The situation with the treatment of resistant RA in current rheumatological practice is different: the use of modern therapies has led to groups of patients with little or no true active inflammation but high DAS scores ${ }^{3}$.

In contrast, some patients with inactive disease, as measured by clinical variables, may still have true, clinically significant, disease activity. Studies using musculoskeletal ultrasound have illustrated this clearly. For example, ultrasound demonstrates subclinical active disease even when patients are categorized as being in remission using composite clinical scores ${ }^{7}$. This ultrasound synovitis predicts later adverse outcomes of structural progression $^{8}$ as well as relapse ${ }^{9}$. We therefore clearly need better tools to discern true disease activity that warrants escalation of therapy. But which is the most accurate, most feasible, and cost-effective?

In the current issue of The Journal, Markusse, et al report that the Multibiomarker Disease Activity Score (MBDA) fulfills this need ${ }^{10}$. They used data from the BeST study to show that MBDA at baseline identifies inflammation that may lead to progressive joint damage more accurately than does the DAS ${ }^{10}$. The MBDA is a score calculated from 12 biomarkers; it correlates significantly with clinical disease activity and aims to better assess RA activity ${ }^{11}$.

The information supplied by more novel tools such as ultrasound and the MBDA is not wholly unique. Of the components of the DAS or DAS28, inflammatory markers and swollen joint counts are associated with ultrasound abnormality, while tender joint count and patient VAS are not ${ }^{12}$. However, by directly measuring inflammation in individual joints, ultrasound can detect low levels of true synovitis that cannot be detected as clinical joint swelling and may be insufficient to result in raised inflammatory markers in the circulation ${ }^{13}$. Hence, ultrasound may provide additional information beyond the DAS or any of its components.

See Predicting radiographic progression in RA, page 2114

Personal non-commercial use only. The Journal of Rheumatology Copyright (c) 2014. All rights reserved. 
The MBDA is based entirely on markers in blood. One of these is the C-reactive protein (CRP), which is probably the best-validated biomarker of inflammation (and subsequent joint damage) in rheumatology, and is also a component of many versions of the DAS. CRP is already known to have value in stratifying risk of radiographic progression and is of course already cheap, convenient, and in widespread use ${ }^{14}$.

The key unanswered question for the MBDA is therefore whether the extra information provided by its wider panel of markers is better able to identify active inflammation (and therefore the risk of radiographic progression) than our existing tools. The information provided by the MBDA over and above CRP is a serum profile of cytokines, growth factors, metalloproteinases, and other related molecules, such as tumor necrosis factor receptor-I, interleukin 6 (IL-6), matrix metalloproteinase-1, leptin, and serum amyloid A. The molecules included in the score all have a degree of face validity owing to their known roles in synovial pathogenesis. However, measurement of cytokines and other soluble mediators in the circulation is notoriously difficult to interpret. Most of these proteins have multiple roles in many tissues in both health and disease, and their local synovial activity in RA may not be reflected by their circulating level. Further, they operate as part of a complex network, with synergy and redundancy, and levels of individual molecules in isolation may oversimplify a complex and heterogeneous disease. Some molecules such as IL-6 are highly correlated with CRP and may therefore provide no additional information. Lastly, cytokines may be no more specific for RA than other inflammatory states, and in this respect have the same limitations as CRP.

The most important study to establish the utility of a tool such as the MBDA would therefore be a comparison of the predictive value of a multibiomarker profile combined with CRP, versus CRP alone, versus neither test. We may find that the existing components of composite outcome measures, such as CRP, already have potential to better predict radiographic progression, but the existing DAS formula and its weightings may not be optimal for this particular purpose in this particular population.

The principle of treating to a target of remission in RA has not diminished in importance in recent years. However, the question of how remission should be defined remains a challenge and is evolving. Demonstrating that a seemingly objective marker of inflammation performs better than the DAS is valuable. But to achieve maximum benefit for people with RA we must weigh the incremental benefit of the measurable variables, and consider which are most feasible and cost effective.

BIANCA DUMITRU, MD; MAYA BUCH, PhD, FRCP;

EDWARD M. VITAL, PhD, MRCP.

National Institute for Health Research (NIHR) Leeds

Biomedical Research Unit,
Leeds Teaching Hospitals National Health Service Trust, Leeds Institute of Rheumatic and Musculoskeletal Medicine, University of Leeds, Leeds, UK.

Address correspondence to Dr. E.M. Vital, Chapel Allerton Hospital, Leeds LS7 4SA, UK; E-mail: e.m.j.vital@leeds.ac.uk

Drs. Vital and Buch are supported by grants from the NIHR.

\section{REFERENCES}

1. Pareto V. Manual of political economy. New York: Augustus M. Kelley Pubs; 1969.

2. Grigor C, Capell H, Stirling A, McMahon AD, Lock P, Vallance R et al. Effect of a treatment strategy of tight control for rheumatoid arthritis (the TICORA study): a single-blind randomised controlled trial. Lancet 2004;364:263-9.

3. Smolen JS, Aletaha D, Bijlsma JW, Breedveld FC, Boumpas D, Burmester G, et al. Treating rheumatoid arthritis to target: recommendations of an international task force. Ann Rheum Dis 2010;69:631-7.

4. Goekoop-Ruiterman YP, de Vries-Bouwstra JK, Allaart CF, van Zeben D, Kerstens PJ, Hazes JM, et al. Clinical and radiographic outcomes of four different treatment strategies in patients with early rheumatoid arthritis (the BeSt study): a randomized, controlled trial. Arthritis Rheum 2005;52:3381-90.

5. Cordingley L, Prajapati R, Plant D, Maskell D, Morgan C, Ali FR, et al. Impact of psychological factors on subjective disease activity assessments in patients with severe rheumatoid arthritis. Arthritis Care Res 2014;66:861-8.

6. Ranzolin A, Brenol JC, Bredemeier M, Guarienti J, Rizzatti M, Feldman D, et al. Association of concomitant fibromyalgia with worse disease activity score in 28 joints, health assessment questionnaire, and short form 36 scores in patients with rheumatoid arthritis. Arthritis Rheum 2009;61:794-800.

7. Foltz V, Gandjbakhch F, Etchepare F, Rosenberg C, Tanguy ML, Rozenberg S, et al. Power Doppler ultrasound, but not low-field magnetic resonance imaging, predicts relapse and radiographic disease progression in rheumatoid arthritis patients with low levels of disease activity. Arthritis Rheum 2012;64:67-76.

8. Brown AK, Conaghan PG, Karim Z, Quinn MA, Ikeda K, Peterfy $\mathrm{CG}$, et al. An explanation for the apparent dissociation between clinical remission and continued structural deterioration in rheumatoid arthritis. Arthritis Rheum 2008;58:2958-67.

9. Saleem B, Brown AK, Quinn M, Karim Z, Hensor EM, Conaghan $\mathrm{P}$, et al. Can flare be predicted in DMARD treated RA patients in remission, and is it important? A cohort study. Ann Rheum Dis 2012;71:1316-21.

10. Markusse IM, Dirven L, van den Broek M, Bijkerk C, Huub Han K, Ronday HK, et al. Multibiomarker Disease Activity Score for rheumatoid arthritis predicts radiographic joint damage in the BeSt Study. J Rheumatol 2014;41:2114-9.

11. Centola M, Cavet G, Shen Y, Ramanujan S, Knowlton N, Swan $\mathrm{KA}$, et al. Development of a multi-biomarker disease activity test for rheumatoid arthritis. PloS One 2013;8:e60635.

12. Hameed B, Pilcher J, Heron C, Kiely PD. The relation between composite ultrasound measures and the DAS28 score, its components and acute phase markers in adult RA. Rheumatology 2008;47:476-80.

13. Saleem B, Brown AK, Keen H, Nizam S, Freeston J, Wakefield R, et al. Should imaging be a component of rheumatoid arthritis remission criteria? A comparison between traditional and modified composite remission scores and imaging assessments. Ann Rheum Dis 2011;70:792-8.

14. Vastesaeger N, Xu S, Aletaha D, St Clair EW, Smolen JS. A pilot risk model for the prediction of rapid radiographic progression in rheumatoid arthritis. Rheumatology 2009;48:1114-21.

J Rheumatol 2014;41:2097-8; doi:10.3899/jrheum.141038 